\title{
Esquisse des types de recherche dans le domaine de l'éducation relative à l'environnement
}

Elizabeth Rooney et Marie Larochelle

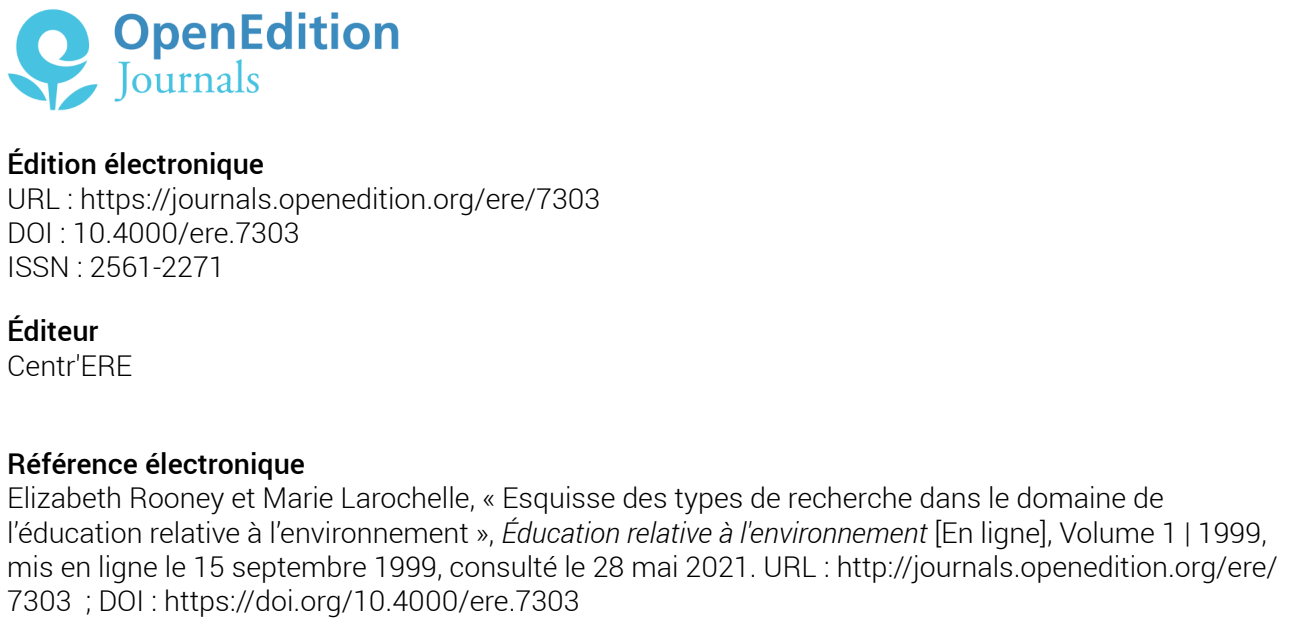

Ce document a été généré automatiquement le 28 mai 2021. 


\title{
Esquisse des types de recherche dans le domaine de l'éducation relative à l'environnement
}

\author{
Elizabeth Rooney et Marie Larochelle
}

L'éducation relative à l'environnement (ERE) existe depuis quelque trente ans à titre d'instrument privilégié pour contribuer à résoudre ce qu'il est convenu d'appeler la crise écologique. Comme tout autre domaine de connaissances et d'intervention, celui de l'ERE n'est pas monolithique et son développement est jalonné par des conceptions et initiatives variées, notamment sur le plan de la recherche. Nous proposons ici une esquisse de ces initiatives, suivant les points de vue qui les animent non seulement à l'égard de ladite crise écologique, mais aussi à l'égard de l'éducation, à savoir : (a) les initiatives de recherche qui souscrivent à l'état de crise que véhicule l'expression " crise écologique», et qui conçoivent celle-ci comme une crise de comportements mal adaptés ; (b) celles qui, tout en souscrivant à cet état de crise, l'envisagent plutôt comme partie prenante d'une crise plus généralisée, à savoir une crise de société; (c) et, enfin, celles qui envisagent ladite crise non pas en termes d'état, mais bien sous l'angle d'une construction sociale. Il va sans dire qu'un tel découpage ne permet pas de rendre justice à toutes les variantes et nuances. C'est donc en gardant à l'esprit cette restriction qu'il faut comprendre nos propos ici qui visent davantage à jeter un éclairage sur le sujet qu'à en proposer une typologie exhaustive ${ }^{1}$.

\section{Une crise de comportements mal adaptés}

Dans la foulée des débats et conférences qui ont marqué la naissance officielle de l'ERE, plusieurs chercheurs, tels Hungerford, Roth ou encore Stapp ${ }^{2}$, se sont intéressés aux attitudes des citoyens et citoyennes à l'égard de la crise dite environnementale et aux moyens de les améliorer en vue de favoriser des comportements plus responsables à l'égard de l'environnement. De manière générale, l'intitulé de cette section encapsule l'orientation donnée à la grande majorité des travaux de ce type : la crise écologique est 
une crise de comportements mal adaptés. Pour rectifier le tir, une attention particulière sera alors accordée au repérage des variables qui sont censées déterminer ces comportements et sur lesquelles il serait possible d'agir en vue de réorienter ces derniers. Plusieurs recherches s'inscriront dans cette orientation dite positiviste réaliste, voire behavioriste, qui a d'ailleurs toujours cours. L'étude récente de Negra et Manning (1997) illustre bien cette option. En effet, en vue de permettre aux gestionnaires d'un parc d'adapter les programmes éducatifs à la clientèle, les auteurs ont dressé une liste des comportements, croyances et valeurs dont feraient preuve les visiteurs, et suggéré des pratiques éducatives différenciées selon les profils ainsi dégagés. Par exemple, pour rejoindre les quelques $42 \%$ de visiteurs dits rationnels, les auteurs recommandent de privilégier des programmes d'interprétation axés sur la gestion rationnelle des ressources, et d'éviter toute référence à caractère religieux. S'appuyant sur le modèle de Hungerford et Volk (1990) et, plus particulièrement, sur l'organigramme des comportements qui le sous-tend, les auteurs lient ainsi de manière causale et linéaire « acquisition de connaissances et modification de comportements ou d'attitudes ». Fruit de nombreuses années de travail, cet organigramme proviendrait de la psychologie sociale des années soixante-dix, années où bien des psychologues soutiennent que leur science peut aider à résoudre la crise environnementale puisque les changements de comportements constitueraient la matière première de la psychologie sociale (voir, par exemple, Maloney et Ward, 1973). Celle-ci permettrait ainsi d'identifier les éléments ou composantes responsables des comportements indésirables ou " mal adaptés », y compris les lacunes sur le plan cognitif.

Pour plusieurs, cette perspective du "déficit cognitif ou comportemental à combler » constituera la voie royale pour contrer les comportements indésirables. En vue de cerner ledit déficit, les pratiques méthodologiques prendront généralement la forme de tests ou de questionnaires standardisés. Les personnes répondantes sont ainsi invitées à se situer à l'égard d'énoncés censés représenter les comportements courants ou souhaités dans le domaine concerné, et à exprimer leur degré d'adhésion sur le sujet. La question qui suit et que nous empruntons à Sivek (1987, p. 161) est exemplaire en ce sens: "Jusqu'à quel point croyez-vous être habile dans l'intervention physique (écogestion) en tant que stratégie d'action environnementale? Aucunement, peu, modérément, considérablement, énormément ». Les matériaux ainsi recueillis feront l'objet d'un traitement quantitatif visant à estimer la robustesse du lien statistique entre l'adhésion exprimée et les variables privilégiées ainsi qu'à identifier, le cas échéant, les éléments pour lesquels une intervention est souhaitable. Le plus souvent, cette intervention relèvera de la même perspective, c'est-à-dire visera à combler le déficit identifié suivant la métaphore typique de la transmission des connaissances, soit la métaphore du conduit qui suppose qu'un émetteur transmet des connaissances qui sont reçues telles quelles par un récepteur.

\section{Une crise de société}

4 Au début des années 1990, des chercheures et chercheurs associés aux universités australiennes et en particulier, au Deakin-Griffith Project, proposent une démarche qui se démarque de l'approche précitée en ce qu'elle serait, au dire de ses protagonistes, de nature plus holistique, critique et participative. Cette démarche s'appuie ainsi sur l'idée que les problèmes environnementaux sont redevables aux systèmes sociaux, 
économiques et politiques actuels, ainsi qu'aux visions du monde, institutions et choix de vie qui les supportent (Fien, 1993, p. v). L'énoncé clé résumant cette approche pourrait être le suivant : la crise environnementale est une crise de société. Dans cette veine, l'accent est mis sur l'implication des organisations à caractère politique à tous les niveaux pour obliger en quelque sorte une relecture des solutions et des correctifs à apporter aux problèmes environnementaux. Un exemple type de la recherche conduite dans cette perspective est l'étude de Greenall Gough et de Robottom (1993). En effet, dans cette étude, l'ERE n'est plus conçue sous le signe du déficit à combler par un ensemble d'éléments conceptuels prescrits. Au contraire, comme en témoigne l'expérience scolaire que les auteurs relatent, elle est envisagée comme une pratique émergente, contextualisée et émancipatrice qui a permis aux élèves de développer une certaine mainmise, un certain pouvoir sur les affaires publiques qui les affectent, telle, dans ce cas, la contamination des plages de leur région.

Caractérisée par une orientation éducative dite socialement critique, cette perspective s'inspire de la conceptualisation que propose Habermas (cité dans Carr et Kemmis, 1986, p. 136) des finalités et intérêts divers qui sous-tendent les connaissances, à savoir :

- l'intérêt technique, axé sur le contrôle et l'exploitation des ressources ;

- l'intérêt pratique ou pragmatique, qui motive la compréhension et la clarification des conditions qui permettent une communication, un dialogue significatif;

- l'intérêt émancipateur, qui enrichit le précédent par l'intégration d'une perspective critique et réflexive sur les cadres et contraintes sociohistoriques de la communication et de l'action sociale, en vue justement de favoriser l'action et l'autonomisation.

Bien que ces intérêts soient le plus souvent imbriqués, c'est l'intérêt émancipateur qui caractériserait plus particulièrement une pédagogie socialement critique, comme l'illustre d'ailleurs l'exemple évoqué plus haut, et qui suppose un partage "maitresélèves » quant à la responsabilité des apprentissages ${ }^{3}$. Les pratiques de recherche privilégiées par cette perspective relèvent de la recherche-action et poursuivent une visée explicite de transformation sociale.

\section{Une construction sociale}

7 Depuis quelques années, une nouvelle tendance émerge quant à la façon d'appréhender la problématique de l'ERE. Des recherches récentes s'intéressent ainsi aux acteurs et actrices non pas en tant qu'individus programmés par des indicateurs observables, mais comme des individus qui cherchent à donner un sens au quotidien, et ce, à l'aide d'une dialectique incessante au sein de leur groupe. Dans cette perspective, l'énoncé clé serait le suivant : la crise environnementale est une affaire de construction sociale. L'étude de Wals (1994) est particulièrement intéressante à cet égard. En effet, on y observe que les personnes se représentent différemment les questions dites environnementales selon leurs communautés d'appartenance. Par exemple, pour des jeunes de quartiers défavorisés dans la région de Détroit, leurs réponses à des questions relatives à ce que représente la nature pour eux, révèlent que si cette dernière peut être un endroit de paix où ils peuvent s'évader à l'occasion des réalités quotidiennes, elle peut aussi être un milieu hostile, les parcs urbains étant souvent vus comme un endroit propice à la criminalité. Pour ces mêmes jeunes, ce qui constitue un enjeu environnemental important est souvent associé à des problèmes de drogues, problèmes auxquels ils sont 
confrontés jour après jour. Comment alors envisager une éducation relative à l'environnement qui tienne compte d'une telle diversité de constructions?

Pour Wals, apprendre est avant tout une affaire de changement conceptuel qui met en jeu non seulement les engagements épistémologiques et les métaphores que soutient la personne qui amorce un tel changement, mais aussi la plausibilité et la viabilité de «la nouvelle conception ». Bien que le modèle de changement conceptuel ait fait l'objet de plusieurs critiques, notamment parce qu'il sous-estime le jeu du cadre épistémique dans la transformation d'une structure conceptuelle (Larochelle et Désautels, 1992, p.60), il suppose des interventions éducatives qui privilégient non pas la transmission d'idées, mais bien la discussion et la confrontation d'idées. En d'autres termes, l'élève ou l'adulte visiteur, par exemple, n'y sont pas vus comme des récepteurs, mais bien comme des constructeurs de connaissances, d'idées, avec lesquelles il faut composer. Sur le plan méthodologique, les outils vont également dans le sens de cette reconnaissance, comme l'illustre le recours fréquent aux techniques d'entretien qui invitent les personnes à exprimer leurs points de vue.

\section{En conclusion}

9 Nous avons proposé, dans les paragraphes qui précèdent, une esquisse des types de recherche effectuée dans le domaine de l'ERE suivant la conception que les recherches véhiculent quant à la crise dite écologique et l'intervention éducative qui s'ensuit. Nous avons ainsi distingué trois axes possibles de regroupement; bien entendu, ce découpage n'est pas exclusif. Par exemple, dans les deux premiers axes, les travaux de recherche s'attachent à repérer les lacunes (individuelles) ou les faiblesses (sociopolitiques) en vue d'y greffer une intervention visant à induire des modifications sur le plan des conduites et solutions en jeu. On pourrait dès lors les envisager comme souscrivant à une même tendance causalisme. Toutefois, là s'arrêtent les similitudes, car les travaux du second axe, tout en maintenant, selon Gough (1993), un rapport aux savoirs encore empreint d'une vision de type positiviste, poursuivent un projet d'émancipation qui est sans commune mesure avec la perspective normative et instrumentale des travaux du premier axe. De même, en faisant place aux enjeux sociopolitiques, ils resocialisent en quelque sorte la question environnementale, rompant ainsi, selon la belle expression d'Abric (1989), avec le "vide expérimental " des travaux du premier axe, et rejoignant à plusieurs égards la thèse mise de l'avant dans le troisième axe.

10 En effet, puisant de nouveau à la psychologie sociale, mais, cette fois, à une psychologie sociale constructiviste, l'approche qui sous-tend les travaux du troisième axe invite à repenser l'agir des personnes comme des conduites situées, c'est-à-dire comme des conduites de construction de sens en lien avec la participation, voire l'appartenance, à un groupe. Issue, entre autres, de la recherche paradigmatique de Moscovici (1961) sur l'image publique de la psychanalyse, cette approche dite «la théorie des représentations sociales " propose ainsi un modèle de la construction des savoirs, de leur manifestation et de leur distribution en liaison avec la société, la culture et les récits qui y sont privilégiés " pour se dire et se penser ». Sur le plan de la recherche, le défi est certes stimulant puisque cette approche, en s'intéressant aux façons par lesquelles les actrices et acteurs sociaux « ordinaires » construisent ce que l'on entend, par exemple, par « problèmes environnementaux », peut donner lieu à l'émergence de 
nouvelles questions et pratiques de recherche. Mais le défi est aussi de taille car, du point de vue méthodologique, ce ne sont plus les "attitudes ou états mentaux » de l'individu qui retiennent l'attention, mais plutôt comment, dans un groupe donné, les uns et les autres mobilisent des façons particulières de "se dire et se penser " et rendent ainsi leur monde intelligible. Autrement dit, dans ce programme qui émerge dans le domaine de l'ERE, les objets de recherche ne relèvent plus d'un monde où il $\mathrm{y} a$ des individus et un environnement, mais bien d'un monde d'actrices et d'acteurs sociaux pour qui il y a un et même, si l'on en croit Wals, des environnements.

\section{BIBLIOGRAPHIE}

Abric, J.-C. (1989). L'étude expérimentale des représentations sociales. Dans D. Jodelet (dir.), Les représentations sociales. Paris : Presses Universitaires de France. p. 187-203.

Carr, W. et Kemmis, S. (1986). Becoming critical : Education, knowledge and action research. London : The Falmer Press.

Fien, J. (1993). Environmental education. A pathway to sustainability. Australia : Deacon University Press.

Gough, N. (1993). Narrative inquiry and critical pragmatism : Liberating research in environmental education. Dans R. Mrazek (dir.), Alternative paradigms in environmental education research. Troy, $\mathrm{OH}$ : The North American Association for Environmental Educa-tion. p. 175-196.

Greenall Gough, A. (1993). Founders in environmental education. Australia : Deakin Uni-versity Press. Greenall Gough, A. et Robottom, I. (1993). Towards a socially critical environmental education : Water quality studies in a coastal school. The Journal of Curriculum Studies, 25(4), 301-316.

Hungerford, H. et Volk, T.L. (1990). Changing learner behavior through environmental education. The Journal of Environmental Education, 21(3), 8-21.

Kelley-Lainé, K. et Posch, P. (1991). Environnement, école et pédagogie active. Paris : Organisation de coopération et de développement économiques (OCDE).

Larochelle, M. et Désautels, J. (1992). Autour de l'idée de science. Itinéraires cognitifs d'étudiants et d'étudiantes. Québec et Bruxelles : Presses de l'Université Laval et De Boeck.

Maloney, M.P. et Ward, M.P. (1973). Ecology : Let's hear from the people. An objective scale for the measurement of ecological attitudes and knowledge. American Psychologist, July, 583-586.

Moscovici, S. (1961). La psychanalyse, son image et son public : étude sur la représentation sociale de la psychanalyse. Paris : Presses Universitaires de France.

Negra, C. et Manning, R.E. (1997). Incorporating environmental behavior, ethics, and values into nonformal environmental education programs. The Journal of Environmental Education, 28(2), 10-21.

Rooney, E. (en cours). L'éducation relative à l'environnement : représentations sociales de praticiens et praticiennes en contexte formel et non formel. Thèse de doctorat en didactique, Université Laval (Québec). 
Sivek, D.J. (1987). An analysis of selected predictors of environmental behavior of three conservation organizations. Dissertation submitted in partial fulfilment of the requirements for the Doctor of Philosophy Degree, Southern Illinois University.

Wals, A.E.J. (1994). Pollution stinks! Young adolescents' perceptions of nature and environ-mental issues with implications for education in urban settings. The Netherlands : Acade-mic Book Center.

\section{NOTES}

1. Pour un point de vue plus détaillé sur la recherche dans le domaine de l'ERE, voir Rooney (en cours).

2. Greenall Gough (1993) situe d'ailleurs ces auteurs parmi les fondateurs de l'ERE

3. C'est en quelque sorte le crédo du Centre pour la recherche et l'innovation dans l'enseignement (CERI) parrainé par l'Organisation de coopération et de développement économiques (OCDE), et dont le programme de support aux initiatives d'ERE en contexte scolaire posait comme condition de participation que les enseignants et enseignantes soient prêts à réfléchir sur leurs actions et à s'engager dans la recherche-action. Voir Kelley-Lainé et Posch (1991).

\section{AUTEURS}

\section{ELIZABETH ROONEY}

Elizabeth Rooney est étudiante au doctorat en didactique à l'Université Laval (Québec). Praticienne durant de nombreuses années dans le domaine de l'éducation relative à l'envi rondement, elle a œuvré dans des contextes tant formels (scolaires) que non formels (centres d'interprétation, parcs). Ses travaux actuels portent sur les représentations sociales qui ont cours dans le domaine.

\section{MARIE LAROCHELLE}

Marie Larochelle est professeure agrégée à l'Université Laval (Québec) et chercheure régulière au Centre interdisciplinaire de recherches sur l'apprentissage et le développement en éducation (CIRADE). Elle s'intéresse plus particulièrement aux jeux et enjeux de la construction des savoirs, y compris des savoirs mobilisés dans l'éducation relative à l'envi rondement. 